\title{
Sistema de ejercicio físico y sugerencia nutricional para los juegos organizados de los educandos de la UNAH-TEC-Danlí
}

Raúl Orlando Figueroa Soriano ${ }^{1}$

\section{RESUMEN}

El presente artículo es el resultado de un estudio experimental (preexperimento, pretest y postest) cuyo objetivo es proponer un sistema de ejercicios físicos y sugerencias de nutrición para mejorar el índice de masa corporal (IMC) y el índice de Ruffier (IR) de forma que se mejore la calidad de vida y se incorpore como eje transversal al programa de la asignatura: Juegos organizados de la UNAH-TECDanlí.

La población es de 70 estudiantes, lo que representa el universo de la indagación, y fue seleccionada de forma intencional. Son varios los autores que han hecho estudios sobre la materia, ya que es de gran importancia para la salud de los educandos; en tal sentido, en este estudio se consideraron fundamentos prestigiosos y a partir de esto se seleccionaron los ejercicios y la sugerencia nutricional para la intervención realizada.

Los ejercicios contribuyeron en gran medida a mejorar el IMC y el IR, puesto que en el primer índice en estudio de la muestra en experimento se mejoró el $84 \%$, se mantuvo el $3 \%$ y empeoró el $13 \%$; en cuanto al segundo índice, con respecto a la muestra en estudio, se mejoró el $95 \%$, se mantuvo el $4 \%$ y empeoró el $1 \%$.

En consecuencia, se obtuvo un 0.99 del coeficiente de Pearson en la correlación IMC e IR, por lo que la propuesta es de gran ayuda para mejorar la calidad de vida, dado que si se mejora el IMC, también se enriquece el funcionamiento del corazón, lo cual se evidenció a través de las técnicas y métodos de investigación aplicada, correspondientes a los niveles empíricos teóricos y matemáticos.

Palabras clave: Sistema de ejercicios, sugerencias de nutrición, índice de masa corporal, índice de Ruffier.

\footnotetext{
${ }^{1}$ Universidad Nacional Autónoma de Honduras. UNAH-TEC-Danlí, Departamento de Humanidades y Artes. Correo electrónico: pitolamaquina2@hotmail.com; pitolamaquina2@yahoo.es.
} 


\section{ABSTRACT}

The article is the result of an experimental study (Pre-experiment [Pre-test and posttest]). Whose aim of this study propose a system of physical exercises and nutrition tips to improve body mass index (BMI) and Ruffier Index (IR) and improve the quality of life, and incorporate it as a central focus of the program organized by subject Games UNAH-TEC-Danlí.

The population is 70 students representing the universe of inquiry and it was selected intentionally. Several authors have done studies on the subject, because of its great importance to the health of students. Prestigious foundations were considered for this study, selecting exercises and nutritional suggestions for the intervention.

Such exercises contributed greatly to improve BMI and IR: the first index of the sample studied in the experiment was improved by $84 \%$, remained still by $3 \%$ and resulted worse in $13 \%$. In the second index relative to the study sample, it was $95 \%$ improved, remained still by $4 \%$ and resulted worse by $1 \%$.

The end result was obtaining a 0.99 Pearson coefficient in BMI and IR correlation. The purpose of this study proves to be helpful in improving the quality of life. If we improve BMI, in turn we improve the functioning of the heart, evidenced through the techniques and methods applied research related to theoretical and mathematical empirical levels.

Keywords: System exercises, nutrition tips, body mass index, index Ruffier.

\section{INTRODUCCIÓN}

La realización regular y sistemática de una actividad física ha demostrado ser una práctica sumamente beneficiosa en la prevención, desarrollo y rehabilitación de la salud, así como un medio para forjar el carácter, la disciplina, la toma de decisiones y el cumplimiento de las reglas beneficiando así el desenvolvimiento del practicante en todos los ámbitos de la vida cotidiana. Actualmente esta visión ha sido aceptada por muchos, sin embargo, a lo largo del tiempo ha tenido sus períodos de auge y regresión.

Aunque la investigación realizada es de naturaleza experimental, está comprometida con la búsqueda de alternativas para tener una calidad de vida óptima, por eso es de gran relevancia en su aplicación, al menos en esta área del conocimiento y 
en UNAH-TEC-Danlí. La indagación consistió en la elaboración de un sistema de ejercicios y sugerencias de nutrición con el propósito de mejorar el índice de masa corporal (IMC) y el índice de Ruffier (IR) con respecto a los parámetros establecidos a nivel nacional e internacional, es decir a la Organización Mundial de la Salud (OMS), ya que durante el proceso docente educativo se evidenciaban anomalías en muchos de los educandos que cursaban la asignatura de Juegos organizados. Este estudio se realizó con alumnos de dicha asignatura de la UNAH-TEC-Danlí y evidenció la mejora de la salud con respecto a una prueba de entrada y una de salida del IMC y el IR, así como de pruebas de laboratorio (hemoglobina) en todo el proceso investigativo.

Para el escudriñamiento se trabajó con una población y muestras representativas que fueron seleccionadas de forma intencional. Se seleccionaron y aplicaron métodos del nivel, empírico, estadístico y teórico a fin de recopilar los datos necesarios, procesarlos cuantitativamente e interpretarlos en función de la toma de decisiones.

La investigación generó un sistema de ejercicio físico y sugerencias de nutrición adaptada a las características de los estudiantes, así como un sistema para generar datos estadísticos para simplificar el proceso de investigación. El resultado hace una contribución a la teoría de la educación física para la salud, demostrando que los fundamentos teóricos referenciales generales de esta teoría, considerados en esta investigación, se cumplen también en el plano particular de esta asignatura y en este contexto, por lo que cobran mayor consistencia y validez como teoría general.

Tratamiento teórico sobre calidad de vida e índice de masa corporal, el índice de Ruffier y hemoglobina

Según Figueroa (2013), el término calidad de vida es el bienestar, felicidad y satisfacción de la persona, que le permite una capacidad de actuación o de funcionar en un momento dado de la vida. Es un concepto subjetivo, propio de cada individuo, que está muy influido por el entorno en el que vive como: la sociedad, la cultura, las escalas de valores.

Según la OMS (2012), la calidad de vida es: "La percepción que un individuo tiene de su lugar en la existencia, en el contexto de la cultura y del sistema de valores en los que vive y en relación con sus objetivos, sus expectativas, sus normas, sus inquietudes. Se trata de un concepto muy amplio que está influido de modo complejo por la salud física del sujeto, su estado psicológico, su nivel de 
independencia, sus relaciones sociales, así como su relación con los elementos esenciales de su entorno".

Atendiendo a esta conceptualización, la OMS propone las dimensiones de la calidad de vida en donde se hace presente la salud física:

1. Dimensión física: es la percepción del estado físico o la salud, entendida como ausencia de enfermedad, los síntomas producidos por la enfermedad y los efectos adversos del tratamiento. No hay duda que estar sano es un elemento esencial para tener una vida con calidad.

2. Dimensión psicológica: es la percepción del individuo de su estado cognitivo y afectivo como el miedo, la ansiedad, la incomunicación, la pérdida de autoestima, la incertidumbre del futuro. También incluye las creencias personales, espirituales y religiosas como el significado de la vida y la actitud ante el sufrimiento.

3. Dimensión social: es la percepción del individuo en las relaciones interpersonales y los roles sociales en la vida como la necesidad de apoyo familiar y social, la relación médico-paciente, el desempeño laboral.

A partir del estudio de calidad de vida se determina en una de sus dimensiones (física) y se hace un análisis del concepto de salud, el cual ha evolucionado mucho a lo largo de los años y de una cultura a otra, es por eso que debe proporcionarse una definición válida para el contexto sociocultural en el que se desenvuelven las personas. Hace unos años se entendía por salud la ausencia de enfermedad, pero esta era una visión reduccionista del propio concepto, además de negativa e incompleta. Actualmente se entiende a la salud como un conjunto de aspectos que engloban situaciones con relación al individuo, a la familia, a la comunidad y todo ello sobre la base de conocimientos, ideas y aspiraciones actuales.

Asimismo, la OMS se refiere a la salud diciendo que es el: "Logro del máximo nivel de bienestar físico, mental y social y de capacidad de funcionamiento que permiten los factores sociales y ambientales en los que vive el sujeto y la colectividad". Como se puede evidenciar, el término calidad de vida está estrechamente ligado al concepto de salud, el cual se determina en gran medida en el primer término enunciado, pues el aspecto salud es uno de los que determina la calidad de vida.

Atendiendo a los conceptos abordados se hará la relación: calidad de vida, salud y actividad física, asumiendo que el individuo debe tomar la responsabilidad personal 
en el cuidado de su propia salud, al mismo tiempo que debe estar consciente de que sus acciones pueden afectar la salud de los que lo rodean y debe sentirse corresponsable en el cuidado de la salud de los que convive; es decir, los objetivos para la educación en los que la cultura física es parte importante.

Entre las acciones preventivas que deben realizarse en las comunidades están las siguientes:

1. Promoción de la salud corporal de las colectividades y comunidades con educación física, recreación y deporte. No solo el individuo es el responsable de su cuidado y corresponsable desde el punto de vista social. Según la OMS (2012), también el Estado tiene una importante responsabilidad.

2. La salud es la mejor palanca para el desarrollo de los pueblos y la mejor plataforma para la paz. Ya no es considerado como un don, sino como un derecho universal y es el Estado el que debe asumir dicha exigencia, pues su logro es una necesidad básica y un objetivo social verdaderamente esencial.

3. La salud y no la enfermedad debe ser el centro de la política sanitaria; al respecto, Piedrola (1996) dice lo siguiente: "Ya nos preocupa tanto el hecho de alargar o prolongar la vida (desde la concepción de salud como la ausencia de enfermedad), sino de aportar calidad a esos años".

Figura 1. Relación calidad de vida, actividad física y salud

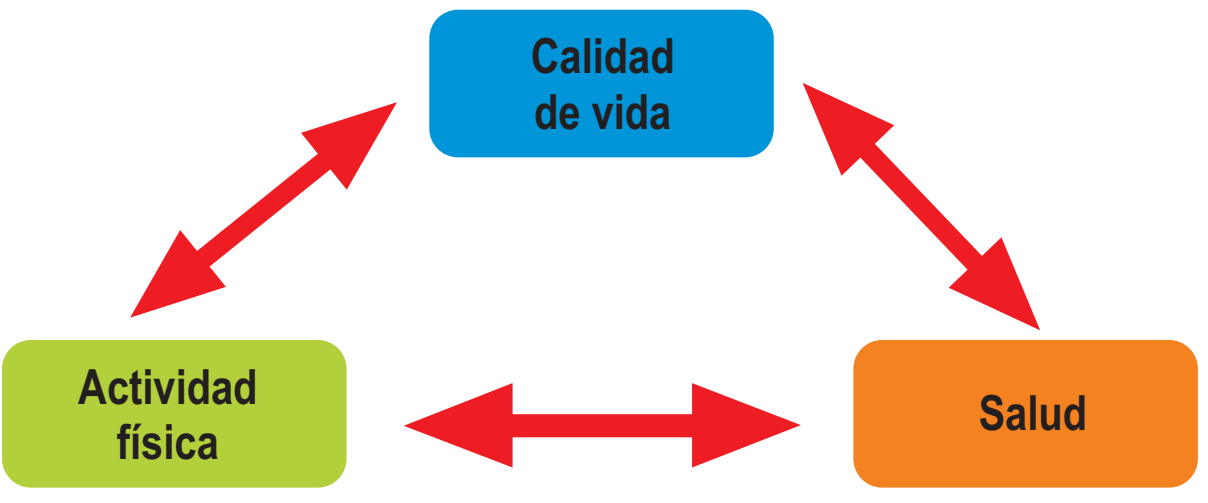

La figura 1 muestra la relación que existe sobre la calidad de vida, la cual a partir de sus dimensiones maneja el estado físico como aspecto primordial para mantener la salud y, a partir de ello, se enuncia que la cultura física busca colaborar en el proceso optimizador de la persona, por tanto intentará aportar beneficios para la salud. 


\section{Efectos del ejercicio sobre diferentes órganos y sistemas}

1. El corazón: el ejercicio físico moderado y continuo favorece en el corazón el aumento de volumen sistólico y del grosor de las paredes cardíacas del ventrículo, dando como resultado una reducción de la frecuencia cardíaca en reposo y de los valores de la tensión arterial. También aumenta la capilarización, incrementando la densidad de los vasos sanguíneos que conectan con el corazón. Estos factores unidos a la reacción metabólica de los lípidos, disminuyen el riesgo de enfermedades coronarias.

Con la edad se produce una disminución de la capacidad contráctil de la musculatura cardíaca, son frecuentes con el paso del tiempo los trastornos eléctricos cardíacos con diferentes grados de bloqueo y arritmia y las válvulas que comunican las distintas cavidades cardíacas se pueden calcificar produciéndose estenosis (estrechamiento) o insuficiencia valvular.

2. El aparato circulatorio: el aumento de la capilarización no solo es un fenómeno que se produce con relación al corazón y los vasos directamente comunicados con él, sino que se produce sobre la mayoría de los vasos que riegan los músculos, órganos y tejidos. Dicho fenómeno ayuda a controlar la tensión arterial reduciendo la aparición de arteriosclerosis y sus consecuencias.

3. Sangre: a nivel venoso, con el envejecimiento, se producirá una disminución de la circulación de retorno, debido a la menor fuerza del efecto bomba que ejercen los músculos de las piernas y a la insuficiencia de las válvulas venosas, por tanto aparecen varices. El ejercicio consigue potenciar los músculos de las piernas bombeando la sangre en su retorno hacia el corazón. A nivel de las arterias, el enemigo más temible es la arteriosclerosis (colesterol); pero el ejercicio físico condiciona a esta enfermedad, ya que se ve reducida la luz de la arteria y con ello el flujo sanguíneo; igualmente, la actividad física favorece la regulación del colesterol sanguíneo y la capilarización, aumentando la irrigación y la oxigenación de los órganos; se observa un aumento de la capacidad de transporte de oxígeno gracias al ascendente número de glóbulos rojos y mayores niveles de concentración de hemoglobina.

4. Sistema respiratorio: con el tiempo va disminuyendo la superficie alveolar y con ello el área destinada al intercambio de gases será menor. Las vías respiratorias, fundamentalmente las de pequeño tamaño, tenderán a la obstrucción, debido a progresos degenerativos a nivel ósteoarticular, la caja torácica se vuelve más rígida. Por todo ello, con el paso del tiempo, se tiene una menor capacidad de adaptación al esfuerzo, debiendo realizar más trabajo para la obtención del oxígeno necesario. En respuesta a estos problemas de salud, con el ejercicio físico se favorece una tonicidad adecuada de la musculatura respiratoria, una optimización del intercambio gaseoso, al igual que una mayor absorción del 
oxígeno de la sangre, una mayor capacidad del volumen respiratorio máximo (VO2), una constante regeneración del volumen residual de aire en los pulmones y se regenera mucho más el volumen residual de los pulmones produciendo una mejor oxigenación.

5. Riñones y vías urinarias: la actividad física favorece una disminución de depósitos en los riñones y conductos urinarios y ayuda a la función de filtro, dado que al hacer ejercicio se eliminan impurezas del organismo por otras vías.

6. Metabolismo: el ejercicio afecta directamente al metabolismo de los lípidos favoreciendo el control de los niveles de colesterol sanguíneo y eliminando tejido graso subcutáneo. De esta forma, el ejercicio participa en el control de la arteriosclerosis y del peso, aspectos responsables de muchas enfermedades.

7. Aparato locomotor: por medio del ejercicio se produce un aumento del volumen y mejora de la capacidad contráctil de la musculatura, al mismo tiempo actúa contra las posibles actividades no bilaterales de la vida diaria, compensando y favoreciendo la movilidad global. Todo ello da un mayor control postural y un mantenimiento de la flexibilidad, ya que el ejercicio moderado afecta a la calcificación de los huesos evitando procesos de osteoporosis.

Con la edad disminuye la velocidad de la contracción muscular y aparece la atrofia de las fibras que componen los músculos, hay una pérdida progresiva de masa muscular y el envejecimiento conlleva, en menor o mayor grado, a una pérdida de la mineralización ósea (osteoporosis). Si bien existe una causa interna debido a la propia senescencia (envejecimiento de los tejidos), la alimentación incorrecta, aporte insuficiente de calcio y sobre todo la falta de ejercicio físico, acelerarán y empeorarán el proceso osteoporótico, con lo cual los huesos serán más frágiles y el riesgo de fractura será mucho mayor. A nivel articular, los cambios degenerativos y la falta de uso supondrán limitaciones para la movilidad, lo cual probablemente conducirá a una progresiva anquilosis de difícil resolución.

Los beneficios que el ejercicio físico supone para el mejor control de la osteoporosis, justificarán por sí solos el inicio de un programa de actividades físicas. El tratamiento farmacológico de la osteoporosis es de eficacia dudosa si no va acompañado de un plan regular de actividad física. El ejercicio evita la temprana atrofia muscular, favorece la movilidad articular y evita la descalcificación ósea.

8. Sistema nervioso: incide positivamente sobre la coordinación, el estado de ánimo, el ritmo de vigilia y sueño, ayudando a prevenir depresiones y a superar situaciones estresantes. Todos los beneficios que trae como consecuencia la actividad física sistemática en los diferentes sistemas y órganos, conlleva a una vida saludable y, por ende, a una mejor calidad de vida. Para realizar una investigación de carácter experimental se debe aplicar un pretest y un postest 
con pruebas validadas que generen datos valiosos. Por ello, se han seleccionado las pruebas de índice de masa corporal y de Ruffier, las cuales se describen a continuación.

a. Índice de masa corporal (IMC)

Determina la masa adecuada de los sujetos y su grado de obesidad, si la hay. Su objetivo radica en valorar la aceptabilidad o línea de normalidad con la relación a la masa o peso y la estatura, el sobrepeso y la obesidad, así como percibir ciertos grados de mal nutrición (ver cuadro 1). Para obtener el IMC se aplica la siguiente fórmula:

$$
\begin{gathered}
\mathrm{IMC}=\mathrm{M}(\mathrm{kg}) \\
(\text { Talla } \mathrm{m})^{2}
\end{gathered}
$$

Cuadro 1. Valores de los rangos del IMC

\begin{tabular}{|l|c|l|}
\hline \multicolumn{1}{|c|}{ Clasificación IMC } & $\mathrm{Kg} / \mathrm{m}^{2}$ & Riesgo de comorbilidad \\
\hline Peso bajo & Menos 18.5 & Bajo \\
\hline Rango normal & $18.5-24.9$ & Promedio \\
\hline Preobesidad (sobrepeso) & $25.0-29.9$ & Incrementado \\
\hline Obesidad grado 1 & $30.0-34.9$ & Moderado \\
\hline Obesidad grado 2 & $35.0-39.9$ & Severo \\
\hline Obesidad grado 3 & Más 40.0 & Muy severo \\
\hline
\end{tabular}

El IMC solo no puede predecir el riesgo para la salud, pero la mayoría de los expertos dicen que un índice superior a 30 (obesidad) no es saludable. Sin importar cuál sea el IMC, el ejercicio puede ayudar a reducir el riesgo de cardiopatía y diabetes; sin embargo, hay que tener el punto de vista de un facultativo para iniciar un programa de ejercicios.

Atendiendo a lo planteado y para hacer mejor las mediciones y la aplicación de un sistema de ejercicios, es necesario conocer el estado físico de la población en estudio y para ello se utiliza la prueba de Ruffier la cual se describe a continuación.

b. Índice de Ruffier

Contribuye a valorar la eficiencia cardiaca al esfuerzo y la reacción del sistema neurovegetativo (reacción vagal o vagotónica) ante el esfuerzo, el 
cual depende del nivel de entrenamiento y de la adaptación del sistema cardiovascular a las cargas físicas. La metodología para aplicar la prueba de Ruffier es la siguiente:

1) Registrar el pulso en reposo (antes de la carga).

2) Realizar 30 cuclillas profundas (los brazos al frente, flexión profunda de las piernas) en $30 \mathrm{seg}$. También se aceptan 30 cuclillas en 30 a $45 \mathrm{~s}$.

3) Registrar el pulso en $10 \mathrm{~s}$, inmediatamente al terminar la carga (0 - $10 \mathrm{~s})$ y tomar un segundo pulso al comenzar el primer minuto y al comenzar el segundo minuto. Cabe recalcar que se toma al terminar el minuto después del esfuerzo para tener las pulsaciones después del esfuerzo y se le suman diez para determinar que si el primer pulso que se tomó después del ejercicio es menor que el segundo, quiere decir que hay una mala recuperación (ver cuadro 2).

Para obtener su índice se aplica la siguiente fórmula:

$$
\mathrm{IR}=\operatorname{Pr}+\mathrm{P} 1+\mathrm{P} 2-200
$$

Cuadro 2. Rangos para evidenciar el IR

\begin{tabular}{|l|l|l|}
\hline \multicolumn{1}{|c|}{$\mathbf{0}$} & \multicolumn{1}{|c|}{ Corazón excepcional } & \multicolumn{1}{c|}{ Excelente } \\
\hline 0 a 5 & Corazón robusto, entrenado & Bien \\
\hline 5 a 10 & Corazón común, mejorable & Regular \\
\hline 10 a 15 & Corazón débil & Pobre \\
\hline Más de 15 & Corazón peligrosamente débil & Malo \\
\hline
\end{tabular}

El índice de Ruffier revela aspectos importantes para determinar el estado físico de la población en estudio, aunque para ello sea necesario determinar el grado de hemoglobina que poseen, ya que este será el punto de partida para determinar la oxigenación de la población con respecto al ejercicio y con ellos la producción de ATP (adenosi tri fosfato). Es por esto que se describe este aspecto a continuación.

La hemoglobina es una heteroproteína de la sangre, de masa molecular 64.000 (64 kDa), de color rojo característico, que transporta el oxígeno desde los órganos respiratorios hasta los tejidos, en vertebrados y algunos invertebrados; al interaccionar con el oxígeno toma un color rojo escarlata, que es el color de la sangre arterial y al perder el oxígeno toma un color rojo oscuro, que es el color característico de la sangre venosa (Dogmaro, 2007). 
Los valores de referencia de la hemoglobina varían de acuerdo a cada laboratorio clínico y por eso se especifican al solicitar la prueba. Esto depende de la ubicación del mismo, específicamente en la calidad de las técnicas usadas (ver cuadro 3 ).

Cuadro 3. Máximo y mínimo de hemoglobina en una persona

\begin{tabular}{|l|c|c|}
\hline \multicolumn{3}{|c|}{ Valor de referencia } \\
\hline \multicolumn{1}{|c|}{ Sexo } & Valor mínimo & Valor máximo \\
\hline Hombre & 10 & 14 \\
\hline Mujer & 8 & 12 \\
\hline
\end{tabular}

En los cuadros 4 y 5 se presentan los valores de la hemoglobina que se toman en cuenta cuando se realiza el ejercicio físico (Ceballos, 1997).

Cuadro 4. Márgenes de hemoglobina en hombres para planificar el ejercicio

\begin{tabular}{|c|c|}
\hline Cantidad & Intensidad de trabajo \\
\hline 10 & $45 \%$ \\
\hline $10-12$ & $65 \%$ \\
\hline $12-13$ & $75 \%$ \\
\hline $13-14$ & $100 \%$ \\
\hline
\end{tabular}

Cuadro 5. Márgenes de hemoglobina en mujeres para planificar el ejercicio

\begin{tabular}{|c|c|}
\hline Cantidad de hemoglobina & Intensidad de trabajo \\
\hline 7 & $45 \%$ \\
\hline $8-9$ & $65 \%$ \\
\hline $9-10$ & $75 \%$ \\
\hline $10-12$ & $100 \%$ \\
\hline
\end{tabular}

En la actualidad son muchos los estudio sobre el IMC referidos al planteamiento de la actividad física, sin embargo, no se encuentra en la bibliografía consultada un estudio a nivel internacional y nacional, como el que se realiza en este estudio, en donde se combine la indagación del IMC, el IR y la prueba de laboratorio sobre la hemoglobina, para evidenciar la aplicación de un sistema de ejercicios y sugerencias de nutrición; de igual manera, sobre si este es eficiente. 


\section{Propuesta de sugerencia de nutrición y sistema de ejercicios}

\section{Objetivo}

Mejorar el índice de masa corporal y el índice de Ruffier en los alumnos de la asignatura Juegos organizados de la UNAH-TEC-Danlí.

\section{Ejemplo del día Lunes}

- Desayuno: un vaso de jugo de naranja y tres galletas saladas con jalea.

- Merienda: fruta.

- Almuerzo:comenzar tomando una taza de caldo de sopa de pollo (sopa Maggie), un plato mediano de arroz blanco cocinado con poco aceite, acompañado con una ensalada de zanahorias y pollo a la plancha, de postre una rodaja de sandía o melón.

- Merienda:fruta.

- Cena: un vaso con leche descremada, con un emparedado de pan integral con lechuga, tomate, queso dietético, acompañado de una ensalada de vegetales.

- Merienda: fruta.

\section{Planificación del entrenamiento}

Objetivo: Mejorar el índice de masa corporal y el índice de Ruffier en los alumnos de la asignatura de Juegos organizados de la UNAH-TEC-Danlí.

Esta planificación de entrenamiento está distribuida para trabajar 3 veces a la semana, pero antes de entrar a la ejecución de la misma se debe realizar un pequeño trote de 10 a 15 min. A continuación se presenta la distribución de los ejercicios a realizar:

\section{Sesión 1 de entrenamiento: trabajo aeróbico}

En las primera dos semanas, hacer 30 minutos de trabajo de trote al $70 \%$ de su capacidad, teniendo en cuenta la frecuencia cardíaca máxima. Esos trotes se harán los días lunes, miércoles, viernes y domingos; los restantes días trabajarán con los circuitos y ejercicios que se presentan en la cuadro 6 . 
Cuadro 6. Sesión 2 de entrenamiento: circuito de ejercicios (Martes)

\begin{tabular}{|l|c|c|c|}
\hline \multicolumn{1}{|c|}{ Ejercicios } & Series & Repetición & Desc. \\
\hline $\begin{array}{l}\text { Realizar calentamiento de movili- } \\
\text { dad articular, toma de pulso y un } \\
\text { trote de 10 minutos, estiramientos }\end{array}$ & -- & -- & \\
\hline Cuclillas & 3 & & \\
\hline Planchas o pechadas & 3 & 15 & \\
\hline Trabajo de tríceps & 3 & 15 & \\
\hline Abdominales & 3 & 15 & \\
\hline Trabajo de hombros & 3 & 15 & \\
\hline Saltadas o salto alterno & 3 & 15 & \\
\hline Trabajo de bíceps & 3 & 15 & \\
\hline
\end{tabular}

\section{METODOLOGÍA}

Para determinar y cumplir con el objetivo de investigación, se aplicaron métodos y técnicas de investigación basadas en todo el proceso investigativo, el cual partió de la siguiente hipótesis de trabajo: si se diseña y ejecuta un sistema de ejercicio físico y sugerencias de nutrición como eje transversal de la asignatura Juegos organizados, entonces se mejora, el índice de masa corporal y el índice de Ruffier y, por ende, en la calidad de vida de los alumnos que cursan dicha asignatura.

La población universo de la investigación fue de 70 alumnos y fue de carácter intencional. En la figura 2 se presenta el proceso realizado. A continuación se presentan los resultados de correlación del pretest y postest.

Figura 2. Proceso realizado

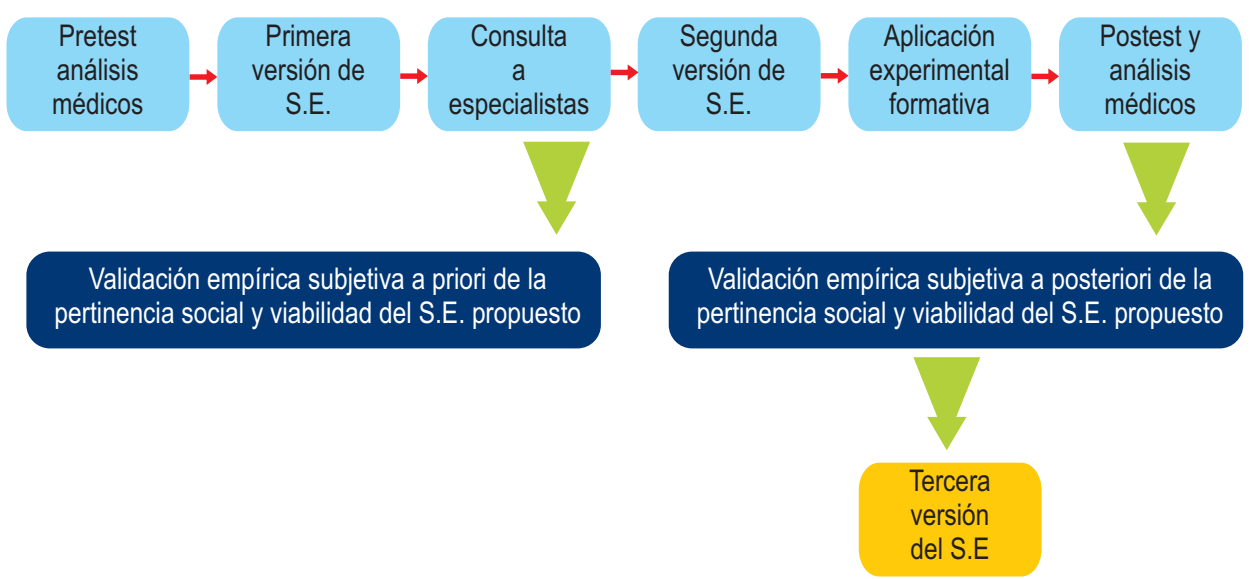


1. Aplicación de un pretest (cuestionario) en el cual se demostró la problemática acerca de la calidad de vida (IMC e IR), así como del análisis de laboratorio.

2. Primera versión del sistema de ejercicios físicos y sugerencias de nutrición.

3. Después de la consulta al especialista, se hizo una segunda versión del sistema de ejercicios físicos y sugerencias de nutrición.

4. Aplicación del sistema de ejercicios físicos y sugerencias de nutrición a través de un preexperimento formativo (experimento cómo método de investigación).

5. Aplicación de un postest (cuestionario) en donde se demostró la problemática acerca de la calidad de vida (IMC e IR), el análisis de laboratorio y cómo a través del ejercicio y las sugerencias de nutrición se puede mejorar dicho parámetro de salud.

Cuadro 7. Comparación de los resultados del cuasi experimento

$$
\text { Índice de masa corporal }
$$

\begin{tabular}{l|l|l|l|l|}
\cline { 2 - 6 } \multicolumn{1}{c}{ Clasificación IMC } & \multicolumn{2}{c|}{ Pretest } & \multicolumn{2}{c|}{ Protest } \\
\hline Peso bajo & Cantidad & $\%$ & \multicolumn{1}{c|}{ Cantidad } & \multicolumn{1}{c|}{$\%$} \\
\hline Rango normal & 13 & 18.57 & 7 & 10 \\
\hline Preobesidad (sobrepeso) & 45 & 64.28 & 51 & 72.85 \\
\hline Obesidad grado 1 & 10 & 14.28 & 10 & 14.28 \\
\hline Obesidad grado 2 & 0 & 0 & 2 & 2.85 \\
\hline Obesidad grado 3 & 1 & 1.42 & 0 & 0 \\
\hline & 1 & 1.42 & 0 & 0 \\
\hline
\end{tabular}

Índice de masa corporal

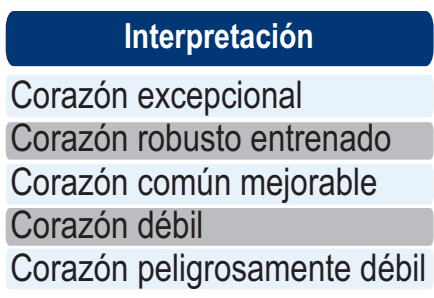

\begin{tabular}{|l|l|l|l|}
\hline \multicolumn{2}{c|}{ Muestra Pretest } & \multicolumn{2}{c|}{ Muestra Protest } \\
\hline Cantidad & $\%$ & \multicolumn{1}{c|}{ Cantidad } & \multicolumn{1}{c|}{$\%$} \\
\hline 0 & 0 & 1 & 1.42 \\
0 & 0 & 0 & 0 \\
\hline 1 & 1.43 & 10 & 14.28 \\
\hline 13 & 18.57 & 30 & 42.86 \\
\hline 56 & 80 & 29 & 41.43 \\
\hline
\end{tabular}


Gráfico 1. Cuasiexperimento en el IMC

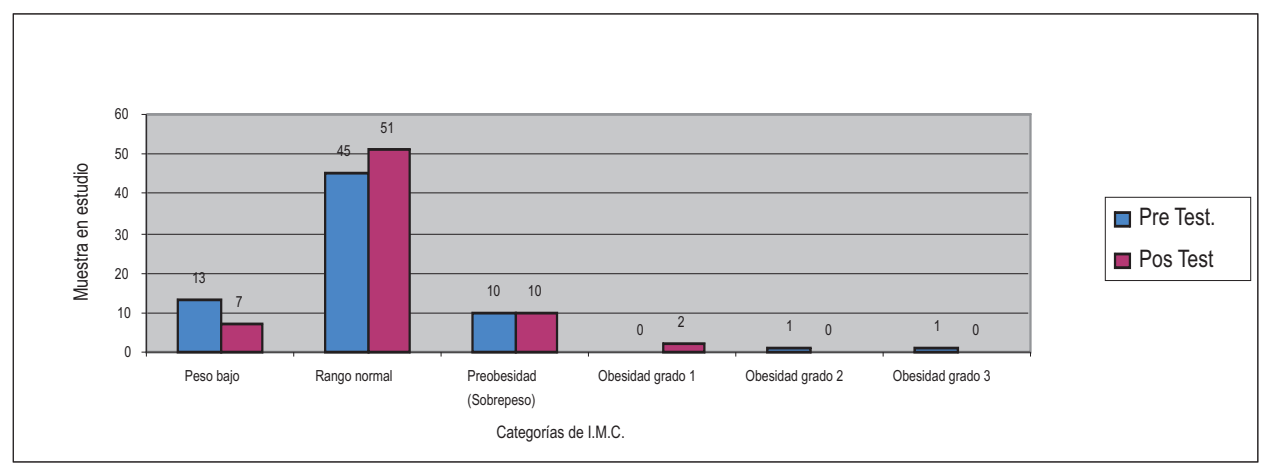

Gráfico 2. Cuasiexperimento en el IR

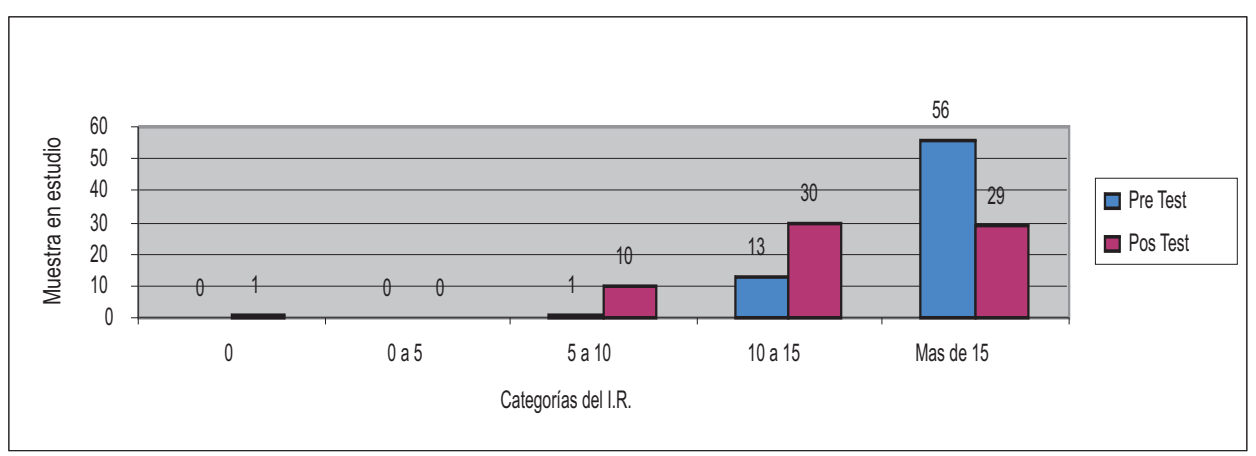

Mediante la aplicación de métodos y técnicas de investigación (análisis médicos, test de Ruffier, observación, consulta a especialistas) se constató el mejoramiento de la población estudiada con respecto al índice de masa corporal y el índice de Ruffier y, por ende, en la calidad de vida de los alumnos que cursan dicha asignatura. Por lo que se comprueba la hipótesis de trabajo planteada. Sustentando aún más esta decisión el coeficiente de correlación Persson que es el 0.99632360. Lo que indica la relación existente entre las dos variables de estudio en lo referente al IMC eIR.

\section{CONCLUSIONES}

1. Los resultados de la sistematización realizada de varios autores especialistas en temas de interés, sobre cultura física y muy especialmente en los referente a la Calidad de Vida y la Educación Física para la salud y en la estructuración de 
sistema de ejercicios físicos y sugerencia de nutrición, permitió determinar que es un aspecto fundamental para el ser humano en correspondencia a la actividad física que desarrolla y su hábito alimenticio.

2. El sistema de ejercicio físico y la sugerencia de nutrición diseñada mediante la combinación de Gimnasia Básica Aeróbica, y la alimentación adecuada, consigue una reducción del peso corporal y (Índice de Masa Corporal y y a su vez la mejora del Índice de Ruffier lo que evidencia la eficiencia cardiaca al esfuerzo y la reacción del sistema neurovegetativo (reacción vagal o vagotónica) ante el esfuerzo, el cuál dependió del nivel de entrenamiento y de la adaptación del sistema cardiovascular a las cargas físicas de la población estudiada.

\section{BIBLIOGRAFÍA}

Alexander, P. (1995). Aptitud física, características morfológicas y composición corporal. Pruebas estandarizadas en Venezuela. Instituto Nacional de Deportes. Caracas: Depoaction.

Alonso, R. (1985). Resultados antropométricos en un ensayo de un nuevo programa de educación física. La Habana: Ciencias Médicas.

Andux, C. (1988). Programa de preparación del deportista de voleibol. La Habana: INDER.

Antón, J. L. (1989). Entrenamiento deportivo en la edad escolar. Bases de aplicación. Málaga: Ed. Junta de Andalucía, UNISPORT.

Barrios, Joaquín y Ranzola Ribas, Alfredo. 1998. Manual para el deporte de iniciación y desarrollo. La Habana: Editorial Deportes.

Baumagnter, T. A. y Jackson, A. S. (1995). Measurement for evaluation in phisical education and exercise science. Madison, Brown \& Benchmark Publishers.

Belbenoi, G. (1973). Le sportàl'école. Tournai: Ed. Casterman.

Berbasco. (1982) Segundo estudio nacional de crecimiento y desarrollo. Revista Cubana de Pediatría. La Habana, 4-22.

Carter, J. L. (1980).The Heath-Carter Somatotype Method. San Diego State University. 